\title{
The Largest Empty Annulus Problem
}

\author{
J. M. Díaz-Báñez ${ }^{1}$, F. Hurtado ${ }^{2}$, H. Meijer ${ }^{3}$, D. Rappaport ${ }^{3}$ and T. \\ Sellares $^{4}$ \\ 1 Universidad de Sevilla, Spain dbanez@cica.es \\ 2 Universitat Politècnica de Catalunya, Spain hurtado@ma2.upc.es \\ 3 Queen's University, Canada henk, daver@cs.queensu.ca \\ 4 Universitat de Girona, Spain sellares@ima.udg.es
}

\begin{abstract}
Given a set of $n$ points $S$ in the Euclidean plane, we address the problem of computing an annulus $A$, (open region between two concentric circles) of largest width such that no point $p \in S$ lies in the interior of $A$. This problem can be considered as a minimax facility location problem for $n$ points such that the facility is a circumference. We give a characterization of the centres of annuli which are locally optimal and we show the the problem can be solved in $O\left(n^{3} \log n\right)$ time and $O(n)$ space. We also consider the case in which the number of points in the inner circle is a fixed value $k$. When $k \in O(n)$ our algorithm runs in $O\left(n^{3} \log n\right)$ time and $O(n)$ space. However if $k$ is small, that is a fixed constant, we can solve the problem in $O(n \log n)$ time and $\mathrm{O}(n)$ space.
\end{abstract}

\section{Introduction}

Consider the placement of an undesirable circular route through a collection of facilities. We assume that the circumference of the circle contains at least one point in its interior. Applications of this problem occur in urban, industrial, military and robotic task planning. For example see [24], [14].

In recent years there has been an increasing interest in considering the location of obnoxious routes (transportation of toxic or obnoxious materials), most of the papers deal with models within an underlying discrete space. For example see $[4,6]$. In the continuous case, in which the route can be located anywhere, there has been very little progress towards obtaining efficient algorithms. An iterative approach for finding the location of a polygonal route which maximizes the minimum distance to a set of points is proposed in [11], but efficient algorithms are not known.

Several problems on computing widest empty corridors have received attention within the area of computational geometry, for example considering empty strips, L-shapes, as well as many other possibilities, see $[8,9$, $16-18,7]$.

These notions can be cast in the setting where an annulus is used for separation. Given a set of points in the plane, we want to separate the set 
into two subsets with the widest annulus. Related results are given in [5], [13], [21] [22].

A dual optimization problem is to find the location of a facility (circumference) which minimizes the maximum distance from all sites. In [10] this problem is termed the sphere-centre problem, and it corresponds to computing the smallest width annulus that contains a given set of points. Efficent algorithms for solving this problem are discussed in [12], [2].

We outline the rest of the paper. In section 2 , we establish some notation and preliminary results. In section 3 , we propose an algorithm to compute the centre of a largest empty annulus. In section 4 , we address a particular case where we fix the number of points in the inner circle of the annulus. Finally, section 5 contains some concluding remarks and poses some open problems.

\section{Characterization of candidate centres}

We begin by introducing some notation. Let $A$ denote an annulus, that is, the open region between two concentric circles. It will be convenient to access features of the annulus, thus we use $c(A)$ to denote the centre of the circles, $r(A)$ and $R(A)$ the radii of the circles, where it understood that $r(A) \leq R(A)$, and $o(A)$ and $O(A)$ the boundary of the circles, such that the radius of $o(A)$ is $r(A)$ and the radius of $O(A)$ is $R(A)$. Let $w(A)=$ $R(A)-r(A)$ denote a quantity we call the width of $A$. We use $d(p, q)$ to denote the Euclidean distance between points $p$ and $q$. Given a set, $S$, of $n$ points in the Euclidean plane, we say that an annulus $A$ is an empty annulus for $S$ if the annulus induces a partition of $S$ into two non-empty subsets $\mathrm{IN}(A, S)=\{s: d(s, c(A)) \leq r(A)\}$ and $\operatorname{OUT}(A, S)=\{s: d(s, c(A)) \geq$ $R(A)\}$. Let $E(S)$ denote the set of all empty annuli for $S$, and let $\Gamma(S)$ denote the subset of $E(S)$ consisting of empty annuli of greatest width, defined precisely as:

$$
\Gamma(S)=\{A \in E(S): w(A) \geq w(B) \text { for all } B \in E(S)\}
$$

Observe that $\Gamma(S)$ is non-empty for any set of two or more points. We define $\omega(S)$ to be equal to $w(A)$ where $A$ is any annulus in $\Gamma(S)$. We present an algorithm that determines the quantity $\omega(S)$. The algorithm will also be able to produce a witness annulus $A \in \Gamma(S)$. Although $\Gamma(S)$ may be infinitely big, our algorithm can also produce a concise description of $\Gamma(S)$. In this section we provide characterizations for largest empty annuli, that is, the annuli in $\Gamma(S)$.

To begin with we make the obvious observation that if $A \in \Gamma(S)$ then

$$
|o(A) \cap S| \geq 1 \text { and }|O(A) \cap S| \geq 1 .
$$




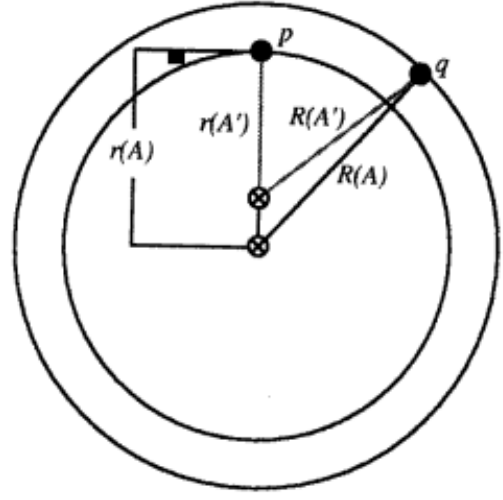

(a)

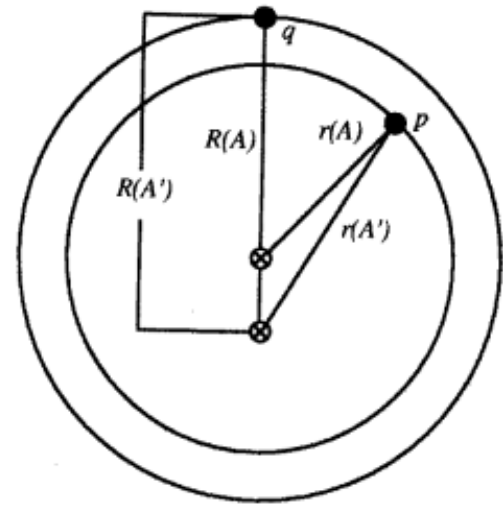

(b)

Fig. 1. If $A$ is an optimal non-syzygy annulus then $|o(A) \cap S| \geq 2$ and $|O(A) \cap S| \geq$ 2 .

Consider the case where an annulus $A \in \Gamma(S)$ has the property that $|\operatorname{IN}(A, S)|=1$. For a point $s \in S$ define $\alpha(s)=\min (d(s, t), t \in S-\{s\})$. In this case $\omega(S)=\max (\alpha(s), s \in S)$. That is $\omega(S)$ is realized by the farthest nearest neighbours. For example if $q$ is the nearest neighbour of $p$ then we can construct an annulus $A$ such that $c(A)=p, r(A)=0$, and $R(A)=d(p, q)$. The all nearest neighbour graph of a set of points is a well known structure that can be found in $\mathrm{O}(n \log n)$ time [20, page 183]. Thus we can easily dispense with this special case.

From now on we restrict our attention to annuli where $|\operatorname{IN}(A, S)|>1$.

A syzygy is an astronomical term meaning the points on the moon's orbit when the moon is in line with the earth and the sun. We borrow this term to define a syzygy annulus as an annulus $A \in \Gamma(S)$ such that there are points $p, q$, with $p \in S \cap o(A)$ and $q \in S \cap O(A)$, and $p$ is contained in the open segment $(c(A), q)$.

Lemma 1. Let $A \in E(S)$, where $A$ is not a syzygy annulus. If $|o(A) \cap S|=$ 1 or $|O(A) \cap S|=1$ then $A \notin \Gamma(S)$.

Proof: We begin by showing that if $A \in E(S)$ such that $|S \cap o(A)|=1$ and $|S \cap O(A)| \geq 1$ then $A \notin \Gamma(S)$. Let $p=S \cap o(A)$. This implies that we can find an annulus $A^{\prime} \in E(S)$ such that $c\left(A^{\prime}\right)$ is on the open segment $\{c(A), p\}$ and there is a $q \in S$ such that $q \in S \cap O(A)$ and $q \in S \cap O\left(A^{\prime}\right)$. See Figure 1 (a). Using the triangle inequality we have:

$$
R\left(A^{\prime}\right)+\left(r(A)-r\left(A^{\prime}\right)\right)>R(A) \rightarrow R\left(A^{\prime}\right)-r\left(A^{\prime}\right)>R(A)-r(A) .
$$


Thus $w\left(A^{\prime}\right)>w(A)$ so $A \notin \Gamma(S)$.

Now suppose that $A \in E(S)$ and $|o(A) \cap S| \geq 2$ and $|O(A) \cap S|=1$. Let $q=S \cap O(A)$. This time we construct an annulus $A^{\prime} \in E(S)$ such that $c(A)$ is on the open segment $\left(c\left(A^{\prime}\right), q\right)$ and there is a $p \in S$ such that $p \in S \cap o(A)$ and $p \in S \cap o\left(A^{\prime}\right)$. See Figure 1 (b). Again by the triangle inequality we have:

$$
r(A)+\left(R\left(A^{\prime}\right)-R(A)\right)>r\left(A^{\prime}\right) \rightarrow R\left(A^{\prime}\right)-r\left(A^{\prime}\right)>R(A)-r(A) .
$$

Thus $w\left(A^{\prime}\right)>w(A)$ so $A \notin \Gamma$.

Thus we conclude that if $A$ is not a syzygy annulus and $|o(A) \cap S|=1$ or $|O(A) \cap S|=1$, then $A \notin \Gamma$.

As a consequence of equation 2 together with lemma 1 we conclude that every optimal non-syzygy annulus $A$ has $|o(A) \cap S| \geq 2$ and $|O(A) \cap S| \geq 2$.

We now deal with the syzygy annuli.

Lemma 2. Suppose that $A$ is a syzygy annulus such that $|\operatorname{IN}(A, S)| \geq 2$ and $A \in \Gamma(S)$. Then there exists an annulus $A^{\prime} \in \Gamma(S)$ such that $\mid o\left(A^{\prime}\right) \cap$ $S \mid \geq 2$.

Proof: If $|o(A) \cap S| \geq 2$, then we set $A^{\prime}=A$ and we are done. Otherwise, using the methods of lemma 1 equation 3 we can obtain an empty annulus $A^{\prime}$ such that $w(A)=w\left(A^{\prime}\right)$ and $\left|o\left(A^{\prime}\right) \cap S\right| \geq 2$.

The preceding lemmas suggest the following theorem.

Theorem 1. If there is an annulus $A \in \Gamma(S)$ such that $|I N(A, S)| \geq 2$ then there is an annulus $A^{\prime} \in \Gamma(S)$ such that $\left|o\left(A^{\prime}\right) \cap S\right| \geq 2$.

Proof: Follows immediately from Lemma 1 and Lemma 2.

This theorem implies that the search space for the centre of a largest empty annulus can be limited to the right bisectors of pairs of points from $S$.

\section{$3 \quad$ Finding a largest empty annulus}

We describe an algorithm to determine the centre of a largest empty annulus that is constrained to lie on the right bisector of a pair of points $p$ and $q$, $B(p, q)$. For convenience we adopt a Cartesian coordinate system such that $B(p, q)$ is the line $L: y=0$. We denote the $x$ and $y$ coordinates of a point $s$ as $x_{s}$ and $y_{s}$. Now for every point $s \in S$ we determine the curve $L_{s}: y=\sqrt{\left(x_{s}-x\right)^{2}+y_{s}^{2}}$. 


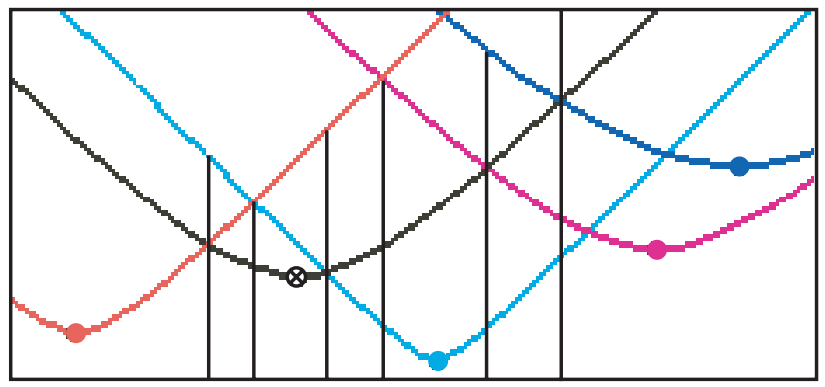

Fig. 2. An arrangement of curves, and a minimization partition of the bisector $B(p, q)$. The point marked with an $\mathrm{x}$ is representative of $p$ and $q$.

Observe that for any two points $s$ and $t$ in $S$, the intersection of $L_{s}$ and $L_{t}$ satisfies

$$
x=\frac{x_{t}^{2}-x_{s}^{2}+y_{t}^{2}-y_{s}^{2}}{2\left(x_{t}-x_{s}\right)}
$$

Thus

- $L_{s}$ and $L_{t}$ are coincident when $x_{s}=x_{t}$ and $y_{s}=-y_{t}$

$-L_{s}$ and $L_{t}$ have no common point when $x_{s}=x_{t}$ and $y_{s} \neq-y_{t}$

- $L_{s}$ and $L_{t}$ have one common point when $x_{s} \neq x_{t}$

For a fixed value of $x$ let $L_{s}(x)=\sqrt{\left(x_{s}-x\right)^{2}+y_{s}^{2}}$. Then

$$
d_{s}(x)= \begin{cases}L_{s}(x)-L_{p}(x) & \text { if } L_{s}(x)>L_{p}(x) \\ \infty & \text { otherwise }\end{cases}
$$

Let $S^{\prime}=S-\{p, q\}$, and set

$$
F(x)=\left\{s \in S^{\prime}: d_{s}(x) \leq d_{t}(x) \text { for all } t \in S^{\prime}\right\} .
$$

Observe that $\mathrm{F}$ induces a partition of $L$ into intervals. Each equivalence class is a maximal interval of $L$ such that for all points in the interval, $F(x)$ is the same subset of $S$. See Figure 2 . We slightly modify the partition such that it only consists of single points and open intervals. Closed intervals of the form $[a, b]$ or a half-open interval $(a, b]$ or $[a, b)$ are replaced by $[a]$, $(a, b),[b]$, or by $(a, b),[b]$, or $[a],(a, b)$ respectively. The number of intervals in the partition is in $\mathrm{O}(n)$, because the partition is a minimization partition of a pseudoline arrangement. See [15].

We can compute the intervals induced by $\mathrm{F}$ in $\mathrm{O}(n \log n)$ time using a divide and conquer algorithm. The merge step simply scans two sorted lists of intervals. We then compute intersections of curves within overlapping intervals to merge in linear time. 
We say that an interval is good if it contains a point $(x, 0)$ such that $d_{s}(x)$ is a positive finite value, for $s \in F(x)$. Then there is an empty annulus $A$ such that $c(A)=(x, 0), p, q \subseteq o(A) \cap S$ and $F(x)=O(A) \cap S$. If $d_{s}(x)=\infty$ for $s \in F(x)$ then a circle centred at $(x, 0)$ and passing through $\{p, q\}$ is a spanning circle of $S$. So this circle is not the inner circle $o(A)$ of an empty annulus $A$.

Once we have a determined the partition of $L$ into intervals, we can determine for each good interval a locally maximal empty annulus. When an interval $u$ is just a single point there is a unique annulus there. Let $u$ be an interval that is not a single point and let $z$ be a value in $u$. Let $s \in F(z)$. We need to find the $x$ such that $d_{s}(x)$ is finite and maximal. It is easy to show that if this maximum exists, then it occurs at $x=\left(x_{s} y_{p}-x_{p} y_{s}\right) /\left(y_{p}-y_{s}\right)$. At this point $x$ the distance between $L_{s}$ and $L_{p}$ is maximal, so either $d_{s}(x)$ is infinite or maximally finite. If $x \in u$ and $d_{s}(x)$ is finite, we have a syzygy annulus $A$ centred at $(x, 0)$ with $\{p, q\} \subseteq o(A) \cap S$ and $F(x)=O(A) \cap S$. If $x \notin u$ then we know that any annulus $A$ centred at a point within the interval $u$ with $p, q \subseteq o(A) \cap S$ and $F(x) \subseteq O(A) \cap S$ is not optimal.

To summarize, by searching through bisectors for every pair of points in $S$, we can determine $\omega(S)$ in $\mathrm{O}\left(n^{3} \log n\right)$ time and $\mathrm{O}(n)$ space. Furthermore we can characterize all annnuli that realize $\omega(S)$ by an ordered pair $(o(A) \cap$ $S, O(A) \cap S)$.

\section{Largest empty $k$-inner points annulus problem}

An interesting related problem fixes the number of points in $\operatorname{IN}(A, S)$. In fact, in some situations we may be interested in separating exactly $k$ points from the rest of the set $S$. Using a separation criteria of the widest circular corridor, leads to the problem of computing a largest empty annulus containing $k$ points in its inner circle.

We adapt the notation of section 2 to handle the situation when the number of points in $\operatorname{IN}(A, S)$ is fixed. Let $E_{k}(S)$ denote the set of all empty annuli with $k$ inner points, and let $\Gamma_{k}(S)$ denote the subset of $E_{k}(S)$ consisting of empty annuli with greatest width.

Theorem 2. If there is an annulus $A \in \Gamma_{k}(S)$ with $k \geq 2$ then there is an annulus $A^{\prime} \in \Gamma_{k}(S)$ such that $\left|o\left(A^{\prime}\right) \cap S\right| \geq 2$.

Proof: The arguments of lemma 1 and lemma 2 do not modify the sets $\operatorname{IN}(A, S)$. Thus the results immediately hold for largest empty $k$-inner point annuli.

We can apply the algorithm of section 3 with a simple modification. Recall, we constrain our search to empty annuli that lie on the right bisector, $B(p, q)$ of a pair of points from $S, p$ and $q$. The simple modification 
is that we only consider empty annuli with $k$-inner points. Consider the arrangement of curves $L_{s}$ for all $s \in S$. For every real value $x$ we define

$$
M(x)=\mid\left\{s \in S: L_{x}(s) \leq L_{x}(p)\right\}
$$

Thus when $M(x)=k$ there is a circle centred at $(x, 0)$ passing through the points $p$ and $q$ and containing exactly $k$ points in its interior and boundary. As before we have a set of intervals. Let us number the intervals from left to right as $I_{0}, I_{1}, \ldots, I_{m}$. If two points $\left(x_{1}, 0\right)$ and $\left(x_{2}, 0\right)$ are both in the same interval then $M\left(x_{1}\right)=M\left(x_{2}\right)$. Furthermore, it is easy to see that we can modify the algorithm that computes the intervals of $L$ in such a way that it also computes $M\left(I_{j+1}\right)-M\left(I_{j}\right)$. Thus we can solve the empty annulus problem when $k$ is fixed in the same time and space bound as before, that is, in $\mathrm{O}\left(n^{3} \log n\right)$ time and $\mathrm{O}(n)$ space.

If $k$ is a small fixed constant, then we can do considerably better by using a different approach. Given a set of points $S$, let $\operatorname{VD}_{k}(S)$ denote the order- $k$ Voronoi diagram of $S$. Recall that $\operatorname{VD}_{k}(S)$ partitions the plane into cells, each of which is a locus of points closest to some $k$ element subset of $S$. See [19]. Let $C$ be a cell in $\operatorname{VD}_{k+1}(S)$ and let $S_{C}$ denote the $k+1$ element subset of $S$ associated with the cell $C$. Suppose $A$ is a largest $k$ inner point empty annulus of $S$ and $c(A)$, the centre of $A$, is in $C$. Then $A$ is also a largest $k$-empty annulus of $S_{C}$ with $\left|\operatorname{IN}\left(A, S_{C}\right)\right|=k$. Moreover, by Lemma 2, at least two points from $S_{C}$ lie on $o(A)$, so $c(A)$ lies on a bisector of two points of $S_{C}$. This leads us to the following algorithm. We first find $\operatorname{VD}_{k+1}(S)$. In [19] D.T. Lee shows that $\operatorname{VD}_{k}(S)$ can be found in $\mathrm{O}\left(k^{2} n \log n\right)$ time and $\mathrm{O}(k(n-k))$ space. For each cell $C$ of $\operatorname{VD}_{k+1}(S)$ we find a point $c(A)$ in $C$ that gives the largest empty $k$-inner point annulus. If $k$ is a small fixed constant, then $\operatorname{VD}_{k+1}(S)$ can be computed in $O(n \log n)$ time and $\mathrm{O}(n)$ space. Finding the largest empty annulus in a cell $C$ can be done in constant time by processing the bisectors of pairs of point from $S_{C}$. Therefore for $k$ a fixed constant we can find the largest empty $k$-inner point annulus in $O(n \log n)$ time and $\mathrm{O}(n)$ space.

\section{Conclusions and further research}

In this paper we have dealt with the problem of computing an empty annulus of maximum width for a set of $n$ points in the plane. We have characterized the centres of annuli which are locally optimal and we have proposed an algorithm to solve the problem in $O\left(n^{3} \log n\right)$ time. We remark that the algorithm is easy to implement and produces a description of all the optimal annuli.

We have also presented an approach by using Voronoi diagrams to solve the case in which the number of interior points is a fixed constant $k$. When $k$ is a fixed constant we obtain a substantially faster algorithm. 
Finally, there are a set of open problems in this context where one may consider parabolic, or conic routes. Then we must find other geometric largest empty regions. Another issue to consider is attempting to find largest empty annuli in higher dimensions.

\section{Acknowledgements}

Ferran Hurtado is partially supported by Projects DGES-MEC PB98-0933, Gen.Cat. SGR1999-0356 and Gen.Cat. SGR2001-0224. Henk Meijer and David Rappaport acknowledge the support of NSERC of Canada research grants. Toni Sellares is partially supported by MEC-DGES-SEUID PB980933 and TIC2001-2392-C03-01 of the Spanish government.

\section{References}

1. P.K. Agarwal And M. Sharir, Daventport-Schinzel sequences and their geometric applications. In Sacks and J.Urrutia, editors, "Handbook of Computational geometry", chapter 1, North Holland, 1999.

2. Agarwal, P.K., Sharir, M. and Toledo S., Applications of parametric searching in geometric optimization. Journal of Algorithms, 17, 1994, 292318.

3. Aurenhammer, F. And Schwarzkopf O., A simple randomized incremental algorithm for computing higher order Voronoi diagrams. In Proc. 7th Annu. Sympos. Comput. Geom., 1995, 142-151.

4. Batta, R. And Chiu, S., Optimal obnoxious paths on a network: Transportation of hazardous materials. Opns. Res., 36, 1988, 84-92.

5. Bhattacharya, B. K. Circular Separability of Planar Point Sets. Computational Morphology, G.T. Toussaint ed. North Holland, 1988.

6. Boffey, B. And Karkazis, J., Optimal location of routes for vehicles: Transporting hazardous materials. European J. Oper. Res., 1995, 201-215.

7. S. Chattopadhyay and P. Das. The $k$-dense corridor problems. Pattern Recogn. Lett., 11:463-469, 1990.

8. S.-W. Cheng. Widest empty corridor with multiple links and right-angle turns. In Proc. 6th Canad. Conf. Comput. Geom., pages 57-62, 1994.

9. S.-W. Cheng. Widest empty L-shaped corridor. Inform. Process. Lett., 58:277-283, 1996.

10. Díaz-Báñez J.M., Mesa J.A. And SchöBel A., Continuous Location of Dimensional Structures. Manuscript.

11. Drezner, Z. And Wesolowsky, G.O., Location of an obnoxious route. Journal Operational Research Society, 40, 1989, 1011-1018.

12. Ebara, H., Nakano, H., Nakanishi, Y. and Sanada, T., A roundness algorithms using the Voronoi diagrams. Transactions IEICE, J70-A 1987, 620-624.

13. Fish S., Separating point sets by circles and the recognition of digital disks. Pattern Analysis and Machine Intelligence, 8 (4), 1986. 
14. Follert, F. Maxmin location of an anchored ray in 3-space and related problems. In 7th Canadian Conference on Computational Geometry, Quebec, 1995.

15. Halperin, D. , Handbook of Discrete and Computational Geometry. Jacob E. Goodman and Joseph O'Rourke eds., CRC Press LLC, Boca Raton, FL, 389-412, 1997.

16. M. Houle and A. Maciel. Finding the widest empty corridor through a set of points. In Snapshots of computational and discrete geometry, pages 201213. Dept. of Computer Science, McGill University, Montreal, Canada, 1988. Technical Report SOCS-88.11.

17. R. Janardan and F. Preparata. Widest-corridor problems. In Proc. 5th Canad. Conf. Comput. Geom., pages 426-431, 1993.

18. R. Janardan and F. P. Preparata. Widest-corridor problems. Nordic J. Comput., 1:231-245, 1994.

19. D. T. Lee. On $k$-nearest neighbor Voronoi diagrams in the plane. IEEE Trans. Comput., vol. C-31, pp. 478487, 1982.

20. O'Rourke J., Computational Geometry in C. Cambridge University Press, 2nd edition, 1998.

21. J. O'Rourke, S.R. Kosaraju and N. Meggido, Computing circular separability. Discrete Computational Geometry, 1 (1), 1986, 105-113.

22. T. J. Rivlin, Approximation by circles. Computing, 21, 93-104 1979

23. M. ShARIR AND P.K. AgARWAL, "Davenport-Schinzel sequences and their geometric applications", Cambridge University Press 1995.

24. Toussaint G. T., Computing largest empty circles with location constraints. International Journal of Computer and Information Sciences, 12, 1983, 347358. 